\title{
Multifaceted role of tocotrienols in cardioprotection supports their structure: function relation
}

\author{
Hannah R. Vasanthi • R. P. Parameswari • \\ Dipak K. Das
}

Received: 24 March 2011/Accepted: 8 April 2011/Published online: 21 May 2011

(c) Springer-Verlag 2011

\begin{abstract}
Tocotrienols are a class of vitamin $\mathrm{E}$ which modulates several mechanisms associated with cardioprotection, anti-cancer, anti-diabetic, and neuroprotection. Unlike other Vitamin E-like compounds, tocotrienols possess inimitable properties. Quite a lot of studies have determined the cardioprotective abilities of tocotrienols and have been shown to possess novel hypocholesterolemic effects together with an ability to reduce the atherogenic apolipoprotein and lipoprotein plasma levels. In addition, tocotrienol has been suggested to have an antioxidant, anti-thrombotic, and antitumor effect indicating that tocotrienol may serve as an effective agent in the prevention and/or treatment of cardiovascular disease and cancer. The bioactivity exhibited is due to the structural characteristics of tocotrienols. Rich sources of tocotrienols which include rice bran, palm oil, and other edible oils exhibit protective effect against cardiovascular disorders. The conclusions drawn from the early literature that vitamin E group of compounds provides an inevitable role in cardioprotection is sustained in many more recent studies.
\end{abstract}

This paper is part of a special issue entitled "Tocotrienols in health and disease" (Guest editors: Bharat B. Aggarwal and Kalaniti Nesaretnam).

H. R. Vasanthi

Department of Biotechnology, School of Life Sciences,

Pondicherry University, Puducherry, Puducherry, India

R. P. Parameswari

Herbal and Indian Medicine Research Laboratory,

Sri Ramachandra University, Chennai, India

D. K. Das $(\bowtie)$

Cardiovascular Research Center, University of Connecticut

School of Medicine, Farmington, CT 06030-1110, USA

e-mail: DDAS@NEURON.UCHC.EDU
Keywords Tocotrienol - Hypocholesterolemia · Ischemia · Preconditioning - Antioxidant .

Cardioprotection

\section{Introduction}

Vitamins are prominent among natural or endogenous compounds considered to be beneficial to human health. The vitamin E group of compounds is among the most prominent of the vitamins because of their antioxidant and related protective properties. News media coverage of vitamin $\mathrm{E}$ influences the awareness of this essential oilsoluble nutrient: not just by the public but also within the scientific community. Combination of significant investigations with reiterating trials ultimately feat into an enhanced scientific understanding, but "media myths" often are self-referential and hardly get damaged by subsequent study results. The case for vitamin $\mathrm{E}$ as protective against heart disease, cancer, and a number of other conditions not long ago was thought to have been open and shut, with further clinical trials expected merely to dot the i's and cross the t's.

Tocopherols (TCPs) and Tocotrienols (TCTs) ${ }^{3}$ (Fig. 1) comprise the vitamin $\mathrm{E}$ family of antioxidants and are synthesized by plants and other photosynthetic organisms. These molecules consist of a chromanol head group linked to an isoprenoid-derived hydrophobic tail. The aliphatic tail of tocopherols is fully saturated, while the side chain of tocotrienols contains three trans double bonds at $3^{\prime}, 7^{\prime}$, and $11^{\prime}$ positions (Fig. 2). Four different forms of tocopherols and tocotrienols occur in nature and differ by the numbers and positions of methyl groups on the aromatic portion of the chromanol head group. The $\alpha$ form of tocotrienols and tocopherols has three methyl groups, the $\beta$ and $\gamma$ forms 
have two methyl groups, and the $\delta$ form has one methyl group on the aromatic ring. In the case of the $\beta$ and $\gamma$ forms, the methyl groups are at positions 5 and 8 or 7 and 8 , respectively, of the chromanol head group. The $\alpha, \beta, \gamma$, and $\delta$ forms of tocopherols and tocotrienols are often referred to collectively as "tocochromanols" (Hunter and Cahoon 2007) (Fig. 3).

Recently, tocotrienols have recently gained increasing scientific interest due to its eminent antioxidative (Mutalib et al. 2003), anti-hypercholesterolemic (Qureshi et al. 2002), and neuroprotective (Khanna et al. 2003) activities that differs somewhat from those of tocopherols. Further, the potent abilities of tocotrienols to induce cell cycle arrest (Wada et al. 2005), to regulate HMG-CoA reductase (Parker et al. 1993), to activate p53 and caspase-8 (Agarwal et al. 2004; Shah and Sylvester 2004), to suppress adhesion molecules (Theriault et al. 2002), to inhibit nuclear factor- $\kappa \mathrm{B}$ (Ahn et al. 2007), and to down regulate c-Myc and telomerase (Eitsuka et al. 2006) have also been reported. Apart from its various other roles, it appears that tocotrienol-mediated cardioprotection is achieved through the preconditioning-like effect, the best yet devised method of cardioprotection. Hence, tocotrienols are expected to accomplish as an important therapeutic option in pharmacological preconditioning and in cardiovascular system.

\section{Sources of tocotrienols}

Tocotrienols are concentrated in cereal grains (i.e., oat, barley, and rye) and certain vegetable oils to various extents (i.e., palm oil and rice bran oil). Edible oils originating from plants are also rich sources for tocotrienols in various proportions. Palm oil contains a particularly high amount of tocotrienols (up to $0.8 \mathrm{~g} / \mathrm{kg}$ ), mainly consisting of $\gamma$-tocotrienol (46\%) and $\alpha$-tocotrienol (22\%) (Sundram and Gapor 1992). Tan and his coworkers illustrated rice bran, palm, and annatto (90\% delta and $10 \%$ gamma)

\section{(a)}



(b)



Fig. 1 a Structure of Tocopherols (TCPs) and b Structure of Tocotrienols (TCTs) ${ }^{3}$

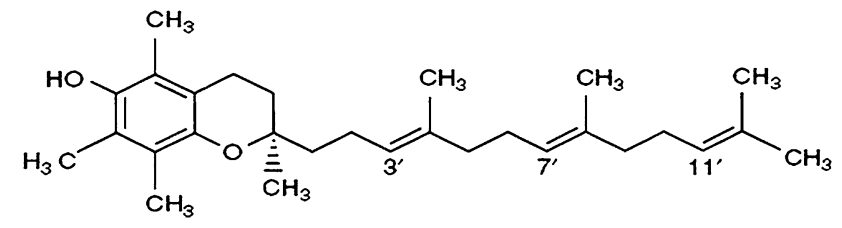

Fig. 2 Tocotrienol with unsaturated side chains at $3^{\prime}, 7^{\prime}$, and $11^{\prime}$ positions
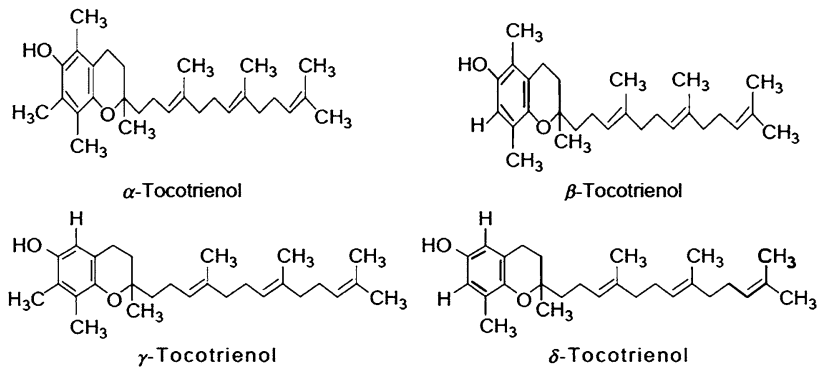

Fig. 3 Biologically Active Isoforms of Tocotrienols

oils as the richest natural sources of tocotrienols. The tocopherols: tocotrienols ratios in rice bran, palm, and annatto oils are 50:50; 25:75; and 0.1:99.9, respectively (Tan 2010). Besides tocopherols, various isomers of tocotrienols have also been detected in human milk (Kobayashi et al. 1975).

\section{Bioavailability of tocotrienols}

The unique biological effects of tocotrienols could be partly explained by their absorption and metabolic fate in vivo. While the absorption mechanisms are fundamentally similar for all vitamin $\mathrm{E}$ compounds, tocotrienols are reported to be absorbed in the digestive tract, incorporated into chylomicrons, and transported in the lymphatic system. It has been reported to be absorbed preferentially into human liver carcinoma (HepG2) cells and degraded to a greater amount (Birringer et al. 2002; Yap et al. 2003). On the other hand, the bioavailability of orally taken tocotrienols is relatively inferior to that of $\alpha$-tocopherol (Sen et al. 2006). Hepatic $\alpha$-Tocopherol transfer protein ( $\alpha$-TTP) is a critical regulator of vitamin $\mathrm{E}$ in mammals, and it selects $\alpha$-tocopherol from tocochromanols in the liver, facilitating $\alpha$-tocopherol secretion into nascent very lowdensity lipoproteins. The presence of a transfer protein that preferentially selects $\alpha$-tocopherol seems to explain why all other forms of vitamin $\mathrm{E}$ have a lower biological activity in the gestation-resorption assay compared with $\alpha$-tocopherol. Even though tocotrienols have a higher radical-scavenging activity than tocopherols, they are less bioavailable after oral ingestion (Hosomi et al. 1997). 
It can be hypothesized that if similar tissue levels could be achieved, tocotrienols would be more effective antioxidants than tocopherols. There is some evidence supporting this hypothesis. When supplementation was carried out in a way that allowed comparable tissue concentrations of $\alpha$-tocopherol and $\alpha$-tocotrienol to be reached in rat microsomes and mitochondria, tocotrienol-supplemented heart tissues were more resistant to lipid peroxidation in vitro than the tocopherol-supplemented counterparts (Serbinova and Packer 1994). However, it is important to note that tocotrienols belong to a family of plant phenolic compounds, which have a brief and transient nature with respect to their metabolism, i.e., compared with $\alpha$-tocopherol, they are inferior with regard to tissue retention and half life. Although affinity between $\alpha$-TPP and tocotrienols is low, it has been shown that tocotrienols orally administered to humans are absorbed from the intestine to the blood, and plasma tocotrienol concentration reaches about $1 \mu \mathrm{M}$ (Fairus et al. 2006). Of note, such circulating level of tocotrienols is almost an order of magnitude higher than that required to protect neurons against a range of neurotoxic insults in cell culture studies (Khanna et al. 2003).

\section{Structure-activity relationship of tocotrienols}

Tocotrienols are natural farnesylated analogs of tocopherols, and the presence of three trans double bonds in the tail distinguishes tocopherols from tocotrienols. The Greek letter prefixes $\alpha, \beta, \gamma$, and $\delta$ designates the degree and placement of methyl substitution on the chroman ring. The naturally occurring tocotrienols possess the (2R), 3'-trans, $7^{\prime}$-trans configuration (Kasparek 1980). The isoforms of tocotrienols differ in their methyl substitution in the chromanol head and possess a 16-carbon hydrocarbon tail; the $\alpha$-isoform contains three methyl groups, whereas the $\beta$ - and $\gamma$-isoforms have two methyl groups, and the $\delta$-isoform has only one methyl group. Tocotrienols have three double bonds, and an unsaturated side chain that renders them greater fluidity and makes them easily accessible to cells with saturated fatty acid layers to be incorporated into cell membranes (Das et al. 2008b). Evidence suggests that the level of phytyl chain saturation and/or chroman ring methylation is critical in determining the differential biopotencies demonstrated by individual vitamin $\mathrm{E}$ isoforms (Crowell et al. 1991; Elson and Yu 1994; McIntyre et al. 2000; Sen et al. 2000). A comparative study between tocotrienols and tocopherols showed that tocotrienols are significantly more potent than tocopherols in most biological actions and display a consistent relationship in most instances corresponding to $\delta$-ocotrienol $\geq \gamma$-tocotrienol $>$ $\alpha$-tocotrienol $>\delta$-tocopherol $>\gamma$-, and $\alpha$-tocopherol (Shukla and Gupta 2005; Sporn and Suh 2002).
Experimental research examining the antioxidant, free radical-scavenging effects of tocopherol and tocotrienols revealed that tocotrienols appear superior due to their better distribution in the fatty layers of the cell membrane and the unsaturated side chain of tocotrienol allows for more efficient penetration into tissues that have saturated fatty layers such as the brain and liver (Suzuki et al. 1993). Yu et al. (2005) evidenced and it was the first study reporting that the polyunsaturated phytol side chain in tocotrienols is involved in its antioxidative activity. The finding says that there are two antioxidative functional groups, the chromanol ring and the polyunsaturated phytol side chain in tocotrienols speaks for being a more potent antioxidant than tocopherols (Brigelius-Flohe and Traber 1999; Packer et al. 2001; Wang and Quinn 1999). This structure-activity relationship indicated that in addition to the requirement of the prenyl side chain for HMG-CoA reductase (HMGR) suppression, changes in the methyl substitution on the chromanol ring may also lead to a divergent effect on HMGR activity (Pearce et al. 1992a). In addition, the chirality at C-2 appears to play a minor role for sterolinhibitory activity.

\section{Antioxidative property of tocotrienols in cardioprotection}

Peroxidation of membrane lipids is known to modify and inactivate cellular components, which can have damaging effects on crucial cellular factors, leading to disease. In the case of LDL-lipids, peroxidation has emerged as the initiating step in the pathogenesis of atherosclerosis. Such modification of LDL causes recognition of the particle by the scavenger cell receptor (i.e., a LDL-receptor-independent pathway) present on macrophages. This pathway is unregulated and nonsaturable, which consequently leads to massive deposition of cholesterol into these cells. This is typically associated with the formation of foam cells in the atherosclerotic lesion (Steinberg et al. 1989). Researchers theorize that antioxidants protect key cell components by neutralizing free radicals before they can cause lipid oxidation or DNA damage. By reducing free radical attack, antioxidants break the chain reaction of lipid peroxidation (chain-breaking antioxidant) and protect the cell membranes by lipid repair and lipid replacement. In this way, they may prevent cancer or cardiovascular disorders. Tocochromanols are the most effective group of lipophilic phenolic antioxidants, and tocotrienols are well recognized for their antioxidative effects. The antioxidant properties of tocotrienols depend primarily on the phenolic group in the chromane ring, rather than the side chain (Burton and Ingold 1989; Niki et al. 1985) and reside mainly with its "chain-breaking" property which neutralizes peroxyl 
radicals generated during lipid peroxidation with the formation of relatively stable phenoxyl radicals (Burton and Traber 1990; Kamal-Eldin and Appelqvist 1996) (Fig. 4).

Nuclear magnetic resonance studies have indicated that $\alpha$-tocotrienol is located closer to the membrane surface, which may facilitate recycling. Furthermore, $\alpha$-tocotrienol has a stronger disordering effect on membranes than $\alpha$-tocopherol and is distributed more uniformly within the membrane. These properties likely enhance the interaction of chromanols with lipid radicals (Serbinova et al. 1993; Suzuki et al. 1993). Tocotrienols show significant inhibition of lipid peroxidation in several model systems and for many years, $\alpha$-tocotrienol was generally considered as the most potent antioxidant against lipid peroxidation in the vitamin $\mathrm{E}$ group. In vivo antioxidant effects of vitamin $\mathrm{E}$ compounds have been studied employing methods such as formation of thiobarbituric acid reactive substances (TBARS) and lipid hydroperoxide (LOOH) (Watkins et al. 1993). Whether antioxidant activity is measured in vitro or in vivo, it is evident that tocotrienols have stronger antioxidant activity than tocopherols (Nesaretnam et al. 1993; Serbinova et al. 1993; Suzuki et al. 1993). Kamat and Devasagayam (1995) in the same study observed, $\alpha$-tocotrienol to be 40 times more effective than $\alpha$-tocopherol in protecting rat liver microsomal membranes against lipid peroxidation and 6.5 times more efficient in protecting cytochrome P-450 from oxidative damage. In the same study, observed similar results in rat brain mitochondria and noted a stronger effect with $\alpha$-tocotrienol. In an elaborate study, a number of mechanisms were shown to contribute to $\alpha$-tocotrienol higher antioxidant activity compared to $\alpha$-tocopherol, including: (a) a more uniform distribution in the membrane lipid bilayer, (b) a more efficient interaction of the chromanol ring with lipid radicals, and (c) a higher recycling efficiency from chromanoxyl radicals (Serbinova et al. 1992).

\section{Lipid-lowering property of tocotrienols in cardioprotection}

High plasma levels of apolipoprotein B-100 (apoB), the protein moiety of LDL is an independent risk factor for the

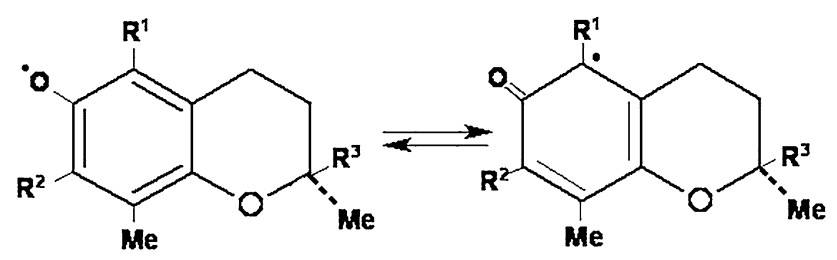

Fig. 4 Formation of stable phenoxyl radicals development of CAD (Albers et al. 1989). Clinical evidence has strongly supported the concept that apoB is a better index of atherogenic risk than total or LDL cholesterol (Sniderman et al. 1980). Lipoprotein (a) is a plasma lipoprotein whose structure resembles that of LDL in which apoB is attached to apoA by a disulfide linkage and their elevated level is also considered atherogenic (Scanu and Fless 1990). The presence of three double bonds in the isoprenoid chain appears to be essential for the inhibition of cholesterogenesis by higher cell penetration evidence for the LDL cholesterol-suppressing potential of tocotrienols (Qureshi et al. 1986). Theriault et al. (1999) studied the effect of $\gamma$-tocotrienol on apoB synthesis, degradation, and secretion in human hepatocellularcarcinoma cell line (HepG2) which revealed that the presence of $\gamma$-tocotrienol in the cells stimulates apoB degradation, and it is speculated that the lack of cholesterol availability reduces the number of secreted apoB-containing lipoprotein particles by limiting translocation of apoB into the endoplasmic reticulum lumen. Several epidemiological studies have shown that tocotrienols reduce the atherogenic apoB plasma levels significantly by $10-15 \%$ (Qureshi et al. 1991a, 1995; Qureshi and Peterson 2001). A case-controlled study (Qureshi et al. 1995) showed that a novel tocotrienol-rich fraction (TRF) from specially processed rice bran oil enriched with didesmethyl-tocotrienol, an isoform of tocotrienols with no methyl groups on the chromanol ring was shown to decrease plasma lipoprotein "a" levels by $17 \%$.

Similarly, hypercholesterolemia is a major contributing factor for the risk of atherosclerosis (Qureshi et al. 1997) and clinical trials have shown that lowering LDL cholesterol with 3-hydroxy-3-methyl-glutarylCoA (HMG-CoA) reductase (HMGR) the rate-limiting enzyme of the cholesterol biosynthetic pathway slows the progression of atherosclerosis and significantly reduces morbidity and mortality from coronary artery disease (CAD) (Pederson 1999; Ross 1999). Tocotrienols influence the mevalonate pathway in mammalian cells by post-transcriptional suppression of HMG-CoA reductase. In elucidating the molecular mechanism for this suppression, the effect was ascribed to the side chain's unique ability to increase cellular farnesol, a mevalonate-derived product, which signals the proteolytic degradation of HMGR (Goldstein and Brown 1990; Rifkind 1998). Side-chain unsaturation is important for the activity of tocotrienols. In vitro models reveal that the intrinsic pharmacological activity of the tocotrienols (rat hepatocytes and HepG2 cell culture) are markedly different and was ascertained that $\gamma$-tocotrienol, $\delta$-tocotrienol, are the only tocotrienols, that lack 5-methyl substitution and are significantly more potent than $\alpha$-tocotrienol in suppressing HMGR. In addition, the chirality at C-2 appears to play a minor role for sterol-inhibitory 
activity (Correll et al. 1994). Qureshi et al. (1996) observed that plasma cholesterol lowering by tocotrienol-rich extracts in chicken and rat was associated with decreased levels of hepatic HMG-CoA reductase activity. Hypercholesterolemic pigs fed the tocotrienol-rich fraction supplement showed a $44 \%$ decrease in total serum cholesterol, a $60 \%$ decrease in LDL cholesterol, and significant decreases in levels of apolipoprotein B $(26 \%)$, thromboxane B2 (41\%), and platelet factor 4 (PF4; 29\%) (Qureshi et al. 1991a). In isolated human liver cells, $\alpha$-tocotrienol $(10 \mathrm{mM}$ for $4 \mathrm{~h})$ inhibited cholesterol synthesis by $32 \%$. Interestingly, $\gamma$ - and $\delta$-tocotrienol, which lacked the 5 -methyl substituents present in $\alpha$-tocotrienol, were shown to possess significantly greater HMGR suppression (Pearce et al. 1994). Tocotrienol not only of palm oil origin but also isolated from rice bran shows cholesterol-lowering properties (Chen and Cheng 2006; Pearce et al. 1992b).

A double-blind, cross-over, 8-wk study was conducted by Quershi et al. (1991b) and his team to compare effects of the tocotrienol-enriched fraction of palm oil $(200 \mathrm{mg}$ palmvitee capsules/day) with those of $300 \mathrm{mg}$ corn oil/d on serum lipids of hypercholesterolemic human subjects (serum cholesterol 6.21-8.02 mmol/L). Serum cholesterol concentrations of seven hypercholesterolemic subjects $(>7.84 \mathrm{mmol} / \mathrm{L}$ ) decreased $31 \%$ during a 4 -wk period in which they were given $200 \mathrm{mg} \gamma$-tocotricnol/d. This indicates that $\gamma$-tocotrienol may be the most potent cholesterol inhibitor in palmvitee capsules.

Lovastatin, a hypocholesterolemic agent useful in the management of hypocholesterolemia, and the tocotrienols have been demonstrated to have cholesterol-lowering properties in animal and humans, but with different mechanisms. A double-blind, cross-over, controlled clinical trial, was carried out in hypercholesterolemic subjects (serum total cholesterol level $>5.7 \mathrm{mmol} / \mathrm{L}$ ) to evaluate the efficacy of therapy: low dose of lovastatin alone $(10 \mathrm{mg} /$ day) or plus a minimum effective dose of tocotrienol mixture $(50 \mathrm{mg} / \mathrm{day})$. The tocotrienol mixture was obtained from rice bran and it is "generally regarded as safe" (GRAS) and has no known side effects, whereas lovastatin has side effects. The study demonstrated that the low dose of tocotrienol mixture, in combined therapy with lovastatin, was an effective reducing cholesterol agent avoiding some adverse effects of statins (Qureshi et al. 2001).

Tocotrienols cause post-transcriptional suppression of HMG-CoA reductase by a process distinct from other known inhibitors of cholesterol biosynthesis (Parker et al. 1993). In vitro, $\gamma$-tocotrienol possesses 30 -fold greater activity toward cholesterol biosynthesis inhibition compared to $\alpha$-tocotrienol. The synthetic (racemic) and natural (chiral) tocotrienols exhibited nearly identical cholesterol biosynthesis inhibition and HMG-CoA reductase suppression properties. Incubation of several cell types with $\gamma$-tocotrienol inhibits the rate of $\left[{ }^{14} \mathrm{C}\right]$ acetate but not $[3 \mathrm{H}]$ mevalonate incorporation into cholesterol in a concentration and time-dependent manner, with $50 \%$ inhibition at $\sim 2 \mathrm{mM}$ and maximum $\sim 80 \%$ inhibition observed within $6 \mathrm{~h}$ in HepG2 cells (Birringer et al. 2002). In vivo studies lend support to in vitro observations (Iqbal et al. 2003). Tocotrienols influence the mevalonate pathway in mammalian cells by post-transcriptional suppression of HMGCoA reductase and specifically modulate the intracellular mechanism for controlled degradation of the reductase protein, an activity that mirrors the actions of the putative nonsterol isoprenoid regulators derived from mevalonate. It is suggested that the farnesyl side chain and the methyl/ hydroxy substitution pattern of $\gamma$-tocotrienol deliver a high level of HMG-CoA reductase suppression, unsurpassed by synthetic analogs studied (Pearce et al. 1994) (Fig. 5).

Ubiquitination followed by rapid degradation by $26 \mathrm{~S}$ proteasomes represents a key mechanism to silence HMG-CoA reductase. This pathway is activated when sterols and nonsterol end products of mevalonate metabolism accumulate in cells. Sterol-accelerated ubiquitination of HMG-CoA reductase requires Insig-1 and Insig-2; membrane-bound proteins of the ER (Sever et al. 2003). Recently, it has been elegantly demonstrated that $\delta$ tocotrienol stimulates the ubiquitination and degradation of HMG-CoA reductase and blocks processing of SREBPs, another sterol-mediated action of Insigs. The $\gamma$ tocotrienol analog was noted to be more selective in enhancing reductase ubiquitination and degradation than blocking the processing of SREBPs. Interestingly, other forms of vitamin $\mathrm{E}$ neither accelerate reductase degradation nor block the processing of SREBPs. $\delta$ - and $\gamma$-tocotrienols trigger reductase ubiquitination directly and do not require further metabolism for their activity (Song and Debose-Boyd 2006).

\section{Anti-atherosclerotic property of tocotrienol in cardioprotection}

Atherosclerosis is a disease of the arterial intima caused by the retention of modified low-density lipoproteins (LDLs)

(6)

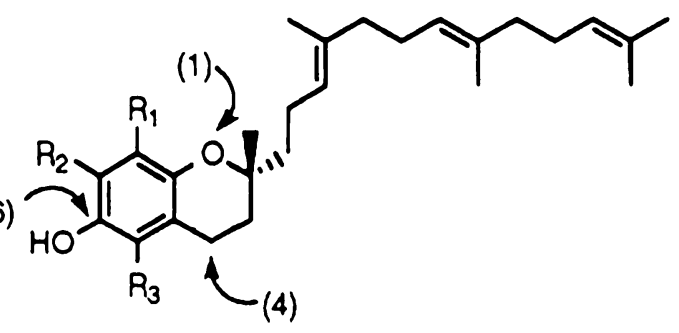

Fig. 5 Lack of 5 Methyl substituent 
and hemodynamic and reductive-oxidative (redox) stress and is characterized by the accumulation of lipids and fibrous elements in the arteries. Endothelial cell injury is the fundamental stimulus responsible for the formation of the atherosclerotic plaque and vascular wall inflammation is a major contributor to the etiology of atherosclerosis (Altman 2004). Tocotrienols differ from tocopherols only in three double bonds in the isoprenoid chain which appear to be essential for the inhibition of cholesterogenesis by higher cell penetration and followed by better interaction with the deposited atherosclerotic plaques (Qureshi et al. 1986). Dietary tocotrienol supplements have been shown to prevent atherosclerosis development in patients and preclinical animal models. One of the most amazing effects of tocotrienol in preventing CVD is its ability to reverse an arterial blockage. A recent study presented data that the tocotrienol-rich fraction of palm oil activated PPAR- $\alpha$, PPAR- $\gamma$, and PPAR- $\delta$ in reporter-based assays. Importantly, tocotrienol-rich fraction diet attenuated the development of atherosclerosis in ApoE-/- mice through inducing PPAR target gene liver $\mathrm{X}$ receptor alpha (LXR alpha) and its down-stream target genes apolipoproteins and cholesterol transporters, suggesting that modulating the activities of PPARs is a key aspect of the in vivo action of tocotrienols ( $\mathrm{Li}$ et al. 2010). In another study by Black et al. (2000), palm tocotrienols reduced lesion formation in atherosclerosis-prone apoE \pm mice by at least two mechanisms. One may have been an antioxidant effect with no alteration in hepatic or serum cholesterol or in serum lipoproteins; the second is largely independent of antioxidant action and may relate to effects of the tocotrienols on foam cell formation and on either hepatic cholesterol secretion or intestinal absorption.

Nafeeza et al. (2001) studied the effect of tocotrienolrich fraction diet on experimentally induced atherosclerosis in the aorta of rabbits which showed a disrupted and fragmented internal elastic lamina in the aorta of rabbits without tocotrienol-rich fraction diet and seemed to be preserved in the group fed with diet rich in tocotrienol. Tocotrienol abridged blood levels of lipid peroxides (measured as TBARS-thiobarbituric acid reactive substances) with an apparent improved blood flow in patients with carotid atherosclerosis. This 3-year double-blind study was conducted at the Jordan Heart Foundation, New Jersey US with 50 patients with a disease condition called carotid stenosis-blockage of the carotid artery, the main artery that supply blood to the brain. Within 6 months of supplementation, $240 \mathrm{mg}$ of palm tocotrienol complex per day, $92 \%$ of the patients had a regression in their carotid stenosis condition. In comparison, none in the placebo patients had any improvement and in fact $4 \%$ of them have had progression in the condition (Tomeo et al. 1995).

\section{Anti-inflammatory role of tocotrienols in cardioprotection}

Inflammation has been found to participate from the initial atherogenesis through lesion progression to the culmination in acute coronary syndromes (plaque rupture) (Libby 2002; Tziakas et al. 2006). Major cellular participants in atherosclerosis include monocytes, macrophages, activated vascular endothelium, T lymphocytes, platelets, and reactive oxygen species. Tocotrienol-rich fraction and its synthesized analog D-P25-T3 have been shown to lower arachidonic acid in various tissues of hereditary hypercholesterolemic swine (Qureshi et al. 2001). It was accounted that there was an overall reduction in prostaglandins and leukotrienes, both of which are synthesized from arachidonic acid, and thus a possible reduction in Interleukin-1. Furthermore, pretreatment with novel tocotrienols has been reported to reduce the induction of Tumor Necrosis Factor (TNF) in response to bacterial lipopolysaccharide (LPS) in mice. The inhibition of TNF by novel tocotrienols thus has been suggested to mitigate atherosclerosis (Qureshi et al. 1993). Pivotal role in atherogenesis is played by macrophages, which are early site for lipid accumulation and mediate the inflammatory and immune response in the intima (Inokuchi et al. 2003; Miyazawa et al. 2004). Napolitano et al. (2007) evaluated the effect of $\alpha$-tocotrienol combined with a new compound FeAOX-6 on macrophage atherogenesis and its related functions and found that the combination induced strong dose-dependent reduction of cholesterol and reduce cholesterol accumulation in human macrophages. The extent of the reduction found with $\alpha$-tocotrienol was greater than that induced by FeAOX-6.

The anti-inflammatory property and mechanism(s) of action of tocotrienol-rich fraction from palm oil was investigated on LPS-induced inflammatory response in human monocytic (THP-1) cells by Wu et al., (2008). It was reported that tocotrienol-rich fraction showed a potent antiinflammatory activity as demonstrated by a dose-dependent inhibition of LPS $(1 \mu \mathrm{g} / \mathrm{mL})$-induced release of NO and PGE2 and a significant decrease in the transcription of proinflammatory cytokines. Tocotrienol-rich fraction at $1 \mu \mathrm{g} / \mathrm{mL}$ significantly blocked the LPS induction of iNOS and COX-2 expression, but not COX-1. This anti-inflammatory activity was further supported by the inhibition of NF- $\kappa \mathrm{B}$ expression. These results conclude that TRF possesses potent anti-inflammatory activity, and its mechanism of action could be through the inhibition of iNOS and $\mathrm{COX}-2$ production, as well as NF- $\kappa \mathrm{B}$ expression.

A recent study by Yam et al. (2009) also supported the anti-inflammatory property of tocotrienol-rich fraction and individual tocotrienol isoforms namely $\delta$-, $\gamma$-, and $\alpha$-tocotrienol on lipopolysaccharide-stimulated RAW264.7 
macrophages. One $\mu \mathrm{g} / \mathrm{mL}$ of tocotrienol-rich fraction and all tocotrienol isoforms significantly inhibited the production of interleukin- 6 and nitric oxide. However, only $\alpha$-tocotrienol demonstrated a significant effect in lowering tumor necrosis factor- $\alpha$ production. Besides, tocotrienolrich fraction and all tocotrienol isoforms except $\gamma$-tocotrienol reduced prostaglandin E2 release. It was accompanied by the down regulation of cyclooxygenase- 2 gene expression by all vitamin $\mathrm{E}$ forms except $\alpha$-tocopherol. Collectively, the data suggested that tocotrienols are better anti-inflammatory agents than $\alpha$-tocopherol and the most effective form is $\delta$-tocotrienol.

\section{Anti-ischemic property of tocotrienols in cardioprotection}

Ischemic heart disease is the most common form of cardiovascular disease (CVD) and is often the underlying cause of angina, acute myocardial infarction (AMI), and heart failure (Lam and Lopaschuk 2007; Stanley et al. 2005; Ussher and Lopaschuk 2009). Myocardial ischemic injury results from severe impairment of coronary blood supply and produces a spectrum of clinical syndromes. Apart from atherosclerotic plaque deposition, oxidative stress is also considered as one of the major causes of ischemic heart disease. The excellent free radical-scavenging property of tocotrienols attenuates the oxidative stress better compared to other vitamin $\mathrm{E}$ analogs and this may be the possible reason for researchers to consider tocotrienols as a better therapeutic option from ischemic heart disease. Although all of the tocotrienol isomers have cardioprotective properties against myocardial ischemic injury, $\gamma$-tocotrienol was the most protective. $\gamma$-tocotrienols are found to act as a myocardial preconditioning agent by activating the eNOS expression (Ikeda et al. 2003). eNOS is considered one of the major cause of intracellular NO generator. This NO then goes on vasodilation and protects the heart from ischemic phase. In another study by Das and his team, it was found that differential interaction of MAPK with caveolin $1 / 3$ in conjunction with proteasome stabilization plays a unique role in tocotrienol-mediated cardioprotection, possibly by altering the availability of prosurvival and anti-survival proteins (Das et al. 2008a). In a study of the cardioprotective properties of $\gamma$-tocotrienol in combination with resveratrol, the two agents acted synergistically, providing a greater degree of cardioprotection than either alone (Lekli et al. 2009). The basis of this effect is their ability to induce autophagy accompanied by activation of Beclin and LC3-II as well as mTOR signaling while simultaneously generating a greater amount of survival signal through activation of the Akt-Bcl-2 survival pathway.
For rat hearts, $\alpha$-tocotrienol was potent in protection against oxidative stress induced by ischemia-reperfusion than $\alpha$-tocopherol (Nafeeza et al. 2001). In another study, tocotrienol-rich fraction was found to inhibit the glutamateinduced pp60 c-Src Kinase activation in HT4 neuronal cells (Crowell et al. 1991). One study indicated that tocotrienol-rich fraction was able to reduce myocardial infarct size, improve postischemic ventricular dysfunction and reduce the incidence of ventricular arrhythmias. Tocotrienol-rich fraction was shown to stabilize $20 \mathrm{~S}$ and $26 \mathrm{~S}$ proteosome activities and reduce the ischemia-reperfusioninduced increase in c-Src phosphorylation (Das et al. 2005). Esterhuyse et al. investigated the effects of red palm oil enriched with tocotrienols and tocopherols on the myocardial nitric oxide-cGMP signaling pathway (Esterhuyse et al. 2005). Treatment with red palm oil increased aortic output and increased levels of cGMP and polyunsaturated fatty acid in rat hearts. Their findings suggest that dietary red palm oil protects via the nitric oxide-cGMP pathway and/or changes in polyunsaturated fatty acid composition during ischemia/reperfusion.

\section{Monocytic adhesion property of tocotrienol in cardioprotection}

The adherence of monocytes to the vascular endothelium is an important early event in atherogenesis. Monocyte adherence to endothelial cells is mediated by multiple cell adhesion molecules, including ICAM-1, VCAM-1, and E-selectin (Carlos and Harlan 1994). Enhanced endothelial expression of these surface adhesion molecules has been shown to be a critical step in foam cell formation and the development of atherosclerosis. Theriault and his co workers studied the potential of tocotrienol for reducing endothelial expression of adhesion molecules and adhesion to monocytes and suggested $\alpha$-tocotrienol to be a more potent inhibitor of adhesion molecule expression and monocytic cell adherence than $\alpha$-tocopherol (Theriault et al. 2002). It was shown that $\alpha$-tocotrienol is effective in reducing monocytic cell adherence. Based on their study, the same group of investigators (Chao et al. 2002) explored efficacy of $\gamma$-tocotrienol versus $\alpha$-tocotrienol on monocyteendothelial cell adhesion and endothelial cell adhesion molecules using HUVEC cell line as the model system. Relative to $\alpha$-tocotrienol, $\gamma$-tocotrienol displayed 1.5 -fold more profound inhibitory effect on monocytic cell adherence using a $15-\mu \mathrm{M}$ concentration within $24 \mathrm{~h}$. This inhibitory action was reversed by coincubation with farnesol and geranylgeraniol, suggesting a role for prenylated proteins in the regulation of monocyte adhesion. The increased intracellular accumulation of $\alpha$-tocotrienol when compared to $\alpha$-tocopherol may also play a role in the higher 
potency of $\alpha$-tocotrienol to inhibit the adhesiveness of endothelial cells (Noguchi et al. 2003). Among the isoforms of tocotrienols, $\delta$-tocotrienols showed to exhibit a highest inhibitory action on monocytic cell adherence (Naito et al. 2005).

\section{Anti-thrombotic property of tocotrienol in cardioprotection}

A thrombus is said to begin when platelets adhere and aggregate to the vascular endothelium forming a fibrous plaque, which can dislodge and obstruct an artery (Hirsh 1987). The anti-thrombotic effect of tocotrienols was demonstrated and was found that tocotrienol-rich fraction and purified $\gamma$-tocotrienol was shown to reduce the synthesis of an eicosanoid, namely thromboxane B2. Aside from thromboxane B2, supplemental tocotrienol was also shown to suppress plasma levels of platelet factor 4 (PF4). The reduction was attributed to reduced activity of phospholipase A2, an enzyme that potentiates the release of arachidonic acid from membrane-bound phospholipids to various eicosanoids (Douglas et al. 1986).

\section{Conclusion}

The ultimate aim of this review is to provide an overview of the multifaceted role of tocotrienols and its isoforms in cardioprotection. Cardiovascular disorder being one of the most potent causes of death throughout the world and the role of tocotrienols in the prevention of it may have significant clinical implications. Out of a minimum of four different isoforms of tocotrienols, $\alpha$ - and $\gamma$-tocotrienols are considered as the effective isoforms which possess the cardioprotective abilities. Collectively, the review raises an intriguing question whether tocotrienols may be further tested in small-scale clinical trials to prevent cardiovascular risks. Outcome studies designed in light of such order would yield lucrative returns.

\section{References}

Agarwal MK, Agarwal ML, Athar M, Gupta S (2004) Tocotrienol rich fraction of palm oil activates $\mathrm{p} 53$, modulates $\mathrm{Bax} / \mathrm{Bcl} 2$ ratio and induces apoptosis independent of cell cycle association. Cell Cycle 3:205-211

Ahn KS, Sethi G, Krishnan K, Aggarwal BB (2007) $\gamma$-Tocotrienol inhibits nuclear factor-kB signaling pathway through inhibition of receptor-interacting protein and TAK1 leading to suppression of antiapoptotic gene products and potentiation of apoptosis. J Biol Chem 282:809-820
Albers JJ, Brunzell JD, Knopp RH (1989) Apoprotein measurements and their clinical application in cholesterol screening. Clin Lab Med 34:4-8

Altman R (2004) Risk factor in coronary atherosclerosis athero-inflammation: the meeting point. Thromb $\mathrm{J}$ 1:4-8

Birringer M, Pfluger P, Kluth D et al (2002) Identities and differences in the metabolism of tocotrienols and tocopherols in HepG2 cells. J Nutr 132:3113-3118

Black TM, Wang P, Maed N et al (2000) Palm Tocotrienols Protect ApoE 1/2 mice from diet-induced atheroma formation. J Nutr 130:2420-2426

Brigelius-Flohe R, Traber MG (1999) Vitamin E: function and metabolism. FASEB J 13:1145-1155

Burton GW, Ingold KU (1989) Vitamin E as in vitro and in vivo antioxidant. Ann N Y Acad Sci 570:7-22

Burton GW, Traber MG (1990) Vitamin E: antioxidant activity, biokinetics and bioavailability. Ann Rev Nut 10:357-382

Carlos TM, Harlan JM (1994) Leukocyte-Endothelial adhesion molecules. Blood 84:2068-2101

Chao JT, Gapor A, Theriault A (2002) Inhibitory effect of deltatocotrienol, a HMG-CoA reductase inhibitor, mononocyteendothelial cell adhesion. J Nutr Sci Vitaminol 48:332-337

Chen CW, Cheng HH (2006) A rice bran oil diet increases LDL receptor and HMG-CoA reductase mRNA expressions and insulin sensitivity in rats with streptozotocin/nicotinamide induced type 2 diabetes. J Nutr 136:1472-1476

Correll CC, Ng L, Edwards PA (1994) Identification of farnesol as the non-sterol derivative of mevalonic acid required for the accelerated degradation of 3-hydroxy-3-methylglutaryl-coenzyme A reductase. J Biol Chem 269:17390-17393

Crowell PL, Chang RR, Ren ZB et al (1991) Selective inhibition of isoprenylation of $21-26-\mathrm{kDa}$ proteins by the anticarcinogen d-limonene and its metabolites. J Biol Chem 266:17679-17685

Das S, Powell SR, Wang P, Divald A, Nesaretnam K, Tosaki A, Cordis GA, Maulik N, Das DK (2005) Cardioprotection with palm tocotrienol: antioxidant activity of tocotrienol is linked with its ability to stabilize proteasomes. Am J Physiol Heart Circ Physiol 289:361-367

Das M, Das S, Wang P, Powell SR, Das DK (2008a) Caveolin and proteasome in tocotrienol mediated myocardial protection. Cell Physiol Biochem 22:287-294

Das S, Lekli I, Das M, Das DK (2008b) Cardioprotection with palm oil tocotrienols: comparision of different isomers. Am J Physiol Heart Circ Physiol 294:970-978

Douglas CE, Chan AC, Choy PC (1986) Vitamin E inhibits platelet phospholipase A2. Biochem Biophys Acta 876:639-645

Eitsuka T, Nakagawa K, Miyazawa T (2006) Down-regulation of telomerase activity in DLD-1 human colorectal adenocarcinoma cells by tocotrienol. Biochem Biophys Res Commun 348:170-175

Elson CE, Yu SG (1994) The chemoprevention of cancer by mevalonate-derived constituents of fruits and vegetables. J Nutr 124:607-614

Esterhuyse AJ, Toit ED, Rooyen JV (2005) Dietary red palm oil supplementation protects against the consequences of global ischemia in the isolated perfused rat heart. Asia Pac J Clin Nutr 14:340-347

Fairus S, Nor RM, Cheng HM, Sundram K (2006) Postprandial metabolic fate of tocotrienol-rich vitamin $\mathrm{E}$ differs significantly from that of $\alpha$ - tocopherol. Am J Clin Nutr 84:835-842

Goldstein JL, Brown MS (1990) Regulation of the mevalonate pathway. Nature 343:425-430

Hirsh J (1987) Hyperactive platelets and complications of coronary artery disease. N Engl J Med 316:1543-1544

Hosomi A, Arita M, Sato Y et al (1997) Affinity for $\alpha$-tocopherol transfer protein as a determinant of the biological activities of vitamin E analogs. FEBS Lett 409:105-108 
Hunter SC, Cahoon EB (2007) Enhancing Vitamin E in oilseeds: unraveling tocopherol and tocotrienol biosynthesis. Lipids 42:97-108

Ikeda S, Tohyama T, Yoshimura $\mathrm{H}$ et al. (2003) Dietary alphatocopherol decreases alpha-tocotrienol but not gamma-tocotrienol concentration in rats. J Nutr 133:428-434

Inokuchi H, Hirokane $\mathrm{H}$, Tsuzuki $\mathrm{T}$ et al (2003) Antiangiogenic activity of tocotrienol. Biosci Biotechnol Biochem 67:1623-1627

Iqbal J, Minhajuddin M, Beg ZH (2003) Suppression of 7, 12-dimethylbenz $[\alpha]$ anthraceneinduced carcinogenesis and hypercholesterolaemia in rats by Tocotrienol rich fraction isolated from rice bran oil. Eur J Cancer Prev 12:447-453

Kamal-Eldin A, Appelqvist LA (1996) The chemistry and antioxidant properties of tocopherols and tocotrienols. Lipids 31:671-701

Kamat J, Devasagayam TPA (1995) Tocotrienols from palm oil as potent inhibitors of lipid peroxidation and protein oxidation in rat brain mitochondria. Neurosci Lett 195:179-182

Kasparek S. (1980). Chemistry of tocopherols and tocotrienols. In Vitamin E: a Comprehensive Treatise-Machlin L. J.Ed Mml Dekker, Inc, New York

Khanna S, Roy S, Ryu H et al (2003) Molecular basis of vitamin E action: tocotrienols modulates 12-lipoxygenase, a key mediator of glutamate induced neurodegeneration. $J$ Biol Chem 278:43508-43515

Kobayashi H, Kanno C, Yamauchi K, Tsugo T (1975) Identification of alpha-, beta gamma-, and delta-tocopherols and their contents in human milk. Biochim Biophys Acta 380:282-290

Lam A, Lopaschuk GD (2007) Anti-anginal effects of partial fatty acid oxidation inhibitors. Curr Opin Pharmacol 7:179-185

Lekli I, Ray D, Mukherjee S, Gurusamy N et al (2009) Coordinated autophagy with resveratrol and gamma-tocotrienol confers synergetic cardioprotection. J Cell Mol Med 14:2506-2518

Li F, Tan W, Kang Z et al (2010) Tocotrienol enriched palm oil prevents atherosclerosis through modulating the activities of peroxisome proliferators-activated receptors. Atherosclerosis 211:278-282

Libby P (2002) Inflammation in atherosclerosis. Nature 420:868-874

McIntyre B, Briski KP, Gapor A, Sylvester PW (2000) Antiproliferative and apoptotic effects tocopherols and Tocotrienols on preneoplastic and neoplastic mouse mammary epithelial cells. Proc Soc Exp Biol Med 224:292-301

Miyazawa T, Inokuchi H, Hirokane H et al (2004) Antiangiogenic potential of tocotrienol in vitro. Biochemistry (Mosc) 69:67-69

Mutalib MSA, Khaza'ai H, Wahle KWJ (2003) Palm-tocotrienol rich fraction (TRF) is a more effective inhibitor of LDL oxidation and endothelial cell lipid peroxidation than alphatocopherol in vitro. Food Res Int 36:405-413

Nafeeza MI, Norzana AG, Jalaluddin HL, Gapor MT (2001) The effects of a tocotrienol-rich fraction on experimentally induced atherosclerosis in the aorta of rabbits. Malays J Pathol 23:17-25

Naito Y, Shimozawa M, Kuroda M et al (2005) Tocotrienols reduce 25 hydroxycholesterol-induced monocyte endothelial cell interactions by inhibiting the surface expression of adhesion molecules. Atherosclerosis 180:19-25

Napolitano M, Avanzi L, Manfredini S, Bravo E (2007) Effects of new combinative antioxidant FeAOX-6 and $\alpha$-tocotrienol on macrophage atherogenesis related functions. Vascular Pharmacol 46:394-405

Nesaretnam K, Devasagayam TPA, Singh BB, Basiron Y (1993) Influence of palm oil or its tocotrienol-rich fraction on lipid peroxidation potential on rat liver mitochondria and microsomes. Biochem Mol Biol Int 30:159-167

Niki E, Kawakami A, Satio M et al (1985) Effect of phytyl side chain of vitamin $\mathrm{E}$ on its antioxidant activity. J Biol Chem 260:2191-2196
Noguchi N, Hanyu R, Nonaka A et al (2003) Inhibition of THP-1 cell adhesion to endothelial cells by alpha-tocopherol and alphatocotrienol is dependent on intracellular concentration of the antioxidants. Free Radic Biol Med 34:1614-1620

Packer L, Weber SU, Rimbach G (2001) Molecular aspects of a-tocotrienol antioxidant action and cell signalling. J Nutr 131:369-373

Parker RA, Pearce BC, Clark RW et al (1993) Tocotrienols regulate cholesterol production in mammalian cells by post-transcriptional suppression of 3-Hydroxy-3-methylglutaryl-coenzyme A reductase. J Biol Chem 268:11230-11238

Pearce BC, Parker RA, Deason ME (1992a) Inhibitors of cholesterol biosynthesis. Hypocholesterolemic and antioxidant activities of benzopyran and tetrahydroaphthalene analogues of the tocotrienols. J Med Chem 37:526-541

Pearce BC, Parker RA, Deason ME et al (1992b) Hypocholesterolemic activity of synthetic and natural tocotrienols. J Med Chem 35:3595-3606

Pearce BC, Parker RA, Deason ME et al (1994) Inhibitors of cholesterol biosynthesis-Hypocholesterolemic and antioxidant activities of Benzopyran and Tetrahydronaphthalene Analogues of the Tocotrienols. J Med Chem 37:526-541

Pederson TR (1999) Statin trials, goals of cholesterol-lowering therapy after AMI. Am Heart J 138:177-182

Qureshi AA, Peterson DM (2001) The combined effects of novel tocotrienols and lovastatin on lipid metabolism in chickens. Atherosclerosis 156:39-47

Qureshi AA, Burger WC, Peterson DM, Elson CE (1986) The structure of an inhibitor of cholesterol biosynthesis isolated from barley. J Biol Chem 261:10544-10550

Qureshi AA, Qureshi N, Halser-Rapacz JO et al (1991a) Dietary tocotrienols reduce concentrations of plasma cholesterol, apolipoprotein $\mathrm{B}$, thromboxane $\mathrm{B} 2$, and platelet factor 4 in pigs with inherited hyperlipidemias. Am J Clin Nutr 53:1042-1046

Qureshi AA, Qureshi N, Wright JfK et al (1991b) Lowering of serum cholesterol in hypercholesterolemic humans by tocotrienols (palmvitee). Am J Clin Nutr 102:1-6

Qureshi N, Hofman J, Qureshi AA (1993) Inhibition of LPS induced tumor necrosis factor synthesis and hypocholesterolermic effect of novel tocotrienols. In "Proceedings of the PORIM International Palm Oil Congress". September 20-25, N16

Qureshi AA, Bradlow BA, Brace L (1995) Response of hypercholesterolemic subjects to administration of tocotrienols. Lipids 30:1171-1177

Qureshi AA, Pearce C, Nor RM et al (1996) Dietary $\delta$-tocopherol attenuates the impact of $\delta$-tocotrienol on hepatic 3-hydroxy-3methylglutaryl coenzyme A reductase activity in chickens. J Nutr 126:389-394

Qureshi AA, Bradlow BA, Salser WA, Brace LD (1997) Novel tocotrienols of rice bran modulate cardiovascular disease risk parameters of hypercholesterolemic humans. Nutr Biochem 8:290-298

Qureshi AA, Peterson DM, Hasler Rapacz JO, Rapacz J (2001a) Novel Tocotrienols of rice bran suppress cholesterogenesis in hereditary hypercholesterolemic swine. J Nutr 131:223-230

Qureshi AA, Sami SA, Salser WA et al (2001b) Synergistic effect of tocotrienol-rich fraction (TRF (25)) of rice bran and lovastatin on lipid parameters in hypercholesterolemic humans. J Nutr Biochem 12:318-329

Qureshi AA, Sami SA, Salser WA, Khan FA (2002) Dose-dependent suppression of serum cholesterol by tocotrienol-rich fraction (TRF25) of rice bran in hypercholesterolemic humans. Atherosclerosis 161:199-207

Rifkind BM (1998) Clinical trials of reducing low-density lipoprotein concentrations. Endocrinol Metab Clin North Am 27:585-595 
Ross R (1999) Atherosclerosis—an inflammatory disease. New Engl J Med 340:115-126

Scanu AM, Fless GM (1990) Lipoprotein (a): heterogeneity and biological relevance. J Clin Invest 86:1709-1715

Sen CK, Khanna S, Roy S, Packer LJ (2000) Molecular basis of Vitamin E action. J Biol Chem 275:13049-13055

Sen CK, Khanna S, Roy S (2006) Tocotrienols: vitamin E beyond tocopherols. Life Sci 78:2088-2098

Serbinova EA, Packer L (1994) Antioxidant properties of alphatocopherol and alpha -tocotrienol. Methods Enzymol 234:354-366

Serbinova E, Khwaja S, Catudioc J et al (1992) Palm oil vitamin E protects against ischemia/reperfusion injury in the isolated perfused Langendorff heart. Nutr Res 12:203-215

Serbinova EA, Tsuchiya M, Goth S, Kagan VE, Packer L (1993) Antioxidant action of a-tocopherol and a-tocotrienol in membranes. In: Vitamin $\mathrm{E}$ in health and disease. Eds. L. Inc., New York, 235-243

Sever N, Song BL, Yabe D et al (2003) Insig-dependent ubiquitination and degradation of mammalian 3-hydroxy-3-methylglutaryl$\mathrm{CoA}$ reductase stimulated by sterols and geranylgeraniol. J Biol Chem 278:52479-52490

Shah S, Sylvester PW (2004) Tocotrienol-induced caspase-8 activation is unrelated to death receptor apoptotic signaling in neoplastic mammary epithelial cells. Exp Biol Med (Maywood) 229:745-755

Shukla S, Gupta S (2005) Dietary agents in the chemoprevention of prostate Cancer. Nutr Cancer 53:18-32

Sniderman A, Shapiro S, Marpole D, Skinner B, Teng B, Kwiterovich PO (1980) Association of coronary atherosclerosis with hyperbetalipoproteinemia [increased protein but normal cholesterol levels in human plasma low-density (beta) lipoproteins]. Proc Natl Acad Sci USA 77:604-608

Song BL, Debose-Boyd RA (2006) Insig-dependent ubiquitination and degradation of 3-hydroxy-3-methylglutaryl coenzyme A reductase stimulated by $\delta$ - and $\gamma$-tocotrienols. J Biol Chem 281:25054-25061

Sporn MB, Suh N (2002) Chemoprevention: an essential approach to controlling cancer. Nat Rev Cancer 2:537-543

Stanley WC, Recchia FA, Lopaschuk GD (2005) Myocardial substrate metabolism in the normal and failing heart. Physiol Rev 85:1093-1129

Steinberg D, Parthasarathy S, Carew TE et al (1989) Beyond cholesterol: modification of lowdensity lipoprotein that increases its atherogenicity. N Engl J Med 320:915-924

Sundram K, Gapor A (1992) Vitamin E from palm oil: its extraction and nutritional properties. Lipid Technol 4:137-141
Suzuki YJ, Tsuchiya M, Wassall SR (1993) Structural and dynamic membrane properties of a-tocopherol and a-tocotrienol: implications to the molecular mechanism of their antioxidant potency. Biochemistry 32:10692-10699

Tan B (2010) Tocotrienols: the New Vitamin E. Spacedocnet, www.spacedoc.net

Theriault A, Wang Q, Gapor A, Adeli K (1999) Effects of $\gamma$-Tocotrienol on ApoB Synthesis, degradation, and secretion in HepG2 Cells. Arterioscler Thromb Vasc Biol 19:704-712

Theriault A, Chao JT, Gapor A (2002) Tocotrienol is the most effective vitamin $\mathrm{E}$ for reducing endothelial expression of adhesion molecules and adhesion to monocytes. Atherosclerosis 160:21-30

Tomeo AC, Geller M, Watkins TR, Gapor A, Bierenbaum ML (1995) Antioxidant effects of tocotrienols in patients with hyperlipidemia and carotid stenosis. Lipids 30:1179-1183

Tziakas DN, Chalikias GK, Kaski JC et al (2006) Inflammatory and anti-inflammatory variable clusters and risk prediction in acute coronary syndrome patients: a factor analysis approach. Atherosclerosis 193:196-203

Ussher JR, Lopaschuk GD (2009) Targeting malonyl CoA inhibition of mitochondrial fatty acid uptake as an approach to treat cardiac ischemia/reperfusion. Basic Res Cardiol 104:203-210

Wada S, Satomi Y, Murakoshi M et al (2005) Tumor suppressive effects of tocotrienol in vivo and in vitro. Cancer Lett 229:181191

Wang X, Quinn PJ (1999) Vitamin E and its function in membranes. Prog Lipid Res 38:309-336

Watkins L, Lenz P, Gapor A, Struck M, Tomeo A, Bierenbaum M (1993) $\gamma$-Tocotrienol as a hypocholesterolemic and antioxidant agent in rats fed atherogenic diets. Lipids 28:1113-1118

Wu SJ, Liu PL, Lean-Teik N (2008) $\gamma$ - Tocotrienol-rich fraction of palm oil exhibits anti-inflammatory property by suppressing the expression of inflammatory mediators in human monocytic cells. Mol Nutr Food Res 52:921-929

Yam ML, Abdul Hafid SR, Cheng HM, Nesaretnam K (2009) Tocotrienols suppress proinflammatory markers and cyclooxygenase-2 expression in RAW264.7 macrophages. Lipids 44:787-797

Yap SP, Yuen KH, Lim AB (2003) Influence of route of administration on the absorption and disposition of $\alpha-, \gamma-$, and $\delta$ - tocotrienols in rats. J Pharm Pharmacol 55:53-58

Yu F-L, Gapor A, Bender W (2005) Evidence for the preventive effect of the polyunsaturated phytol side chain in tocotrienols on $17 \beta$-estradiol epoxidation. Cancer Detect Prev 29:383-388 